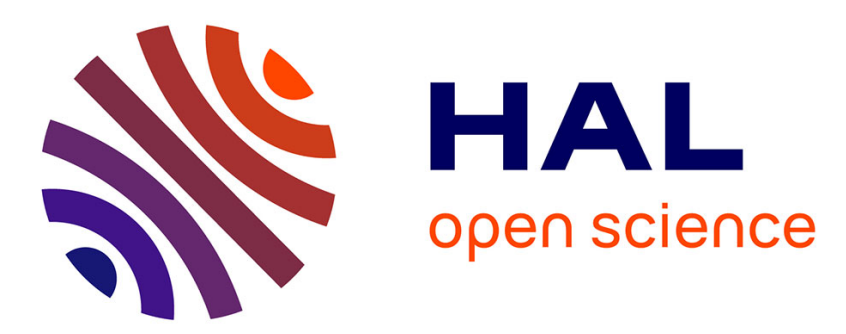

\title{
On pulse solutions of a reaction-diffusion system in population dynamics
}

Vitaly Volpert, Natalia Reinberg, Mohammed Benmir, Soumaya Boujena

\section{To cite this version:}

Vitaly Volpert, Natalia Reinberg, Mohammed Benmir, Soumaya Boujena. On pulse solutions of a reaction-diffusion system in population dynamics. Nonlinear Analysis: Theory, Methods and Applications, 2015, 10.1016/j.na.2015.02.017 . hal-01237675

\section{HAL Id: hal-01237675 \\ https://hal.science/hal-01237675}

Submitted on 8 Dec 2015

HAL is a multi-disciplinary open access archive for the deposit and dissemination of scientific research documents, whether they are published or not. The documents may come from teaching and research institutions in France or abroad, or from public or private research centers.
L'archive ouverte pluridisciplinaire HAL, est destinée au dépôt et à la diffusion de documents scientifiques de niveau recherche, publiés ou non, émanant des établissements d'enseignement et de recherche français ou étrangers, des laboratoires publics ou privés. 


\title{
On pulse solutions of a reaction-diffusion system in population dynamics
}

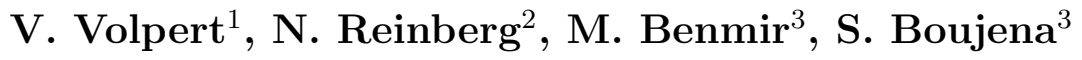 \\ ${ }^{1}$ Institut Camille Jordan, UMR 5208 CNRS, University Lyon 1, 69622 Villeurbanne, France \\ ${ }^{2}$ Institute of Problems of Mechanical Engineering, Russian Academy of Sciences \\ 199178 Saint Petersburg, Russia \\ ${ }^{3}$ Faculté des Sciences, University Hassan II of Casablanca, Casablanca 20100 Maroc
}

\begin{abstract}
A reaction-diffusion system of equation describing the distribution of population density is considered. The existence of pulse solutions is proved by the Leray-Schauder method based on the topological degree for elliptic operators in unbounded domains and on a priori estimates of solutions. Numerical simulations show that such solutions become stable in the case of global consumption of resources while they are unstable without the integral terms. This model is used to describe human height distribution.
\end{abstract}

Key words: reaction-diffusion systems, stationary pulses, Leray-Schauder method, human height distribution

\section{Introduction}

In this work we study the system of equations

$$
\begin{gathered}
\frac{\partial u}{\partial t}=d_{1} \frac{\partial^{2} u}{\partial x^{2}}+a u v(1-r I(u)-r I(v))-b u \\
\frac{\partial v}{\partial t}=d_{2} \frac{\partial^{2} v}{\partial x^{2}}+c u v(1-r I(u)-r I(v))-d v
\end{gathered}
$$

on the whole axis. We will consider its positive stationary solutions decaying at infinity. We will call such solutions pulses. Here all coefficients of the equations are positive constants,

$$
I(u)=\int_{-\infty}^{\infty} u(y, t) d y, \quad I(v)=\int_{-\infty}^{\infty} v(y, t) d y .
$$

In the context of population dynamics, this system describes the distribution of the population in the case of sexual reproduction [7]. Here $u$ is the density of males, $v$ the density 
of females, the space variable $x$ corresponds to their phenotype. Diffusion terms describe small random changes of the phenotype of offsprings in comparison with the phenotype of parents. The reproduction terms are proportional to the product of densities and to available resources $(K-r I(u)-r I(v))$, where the carrying capacity $K=1$ shows the rate of production of resources, while their consumption is proportional to the total population $I(u)+I(v)$. The last terms in the right-hand side of equations (1.1), (1.2) describe mortality of the population.

If we assume that all parameters characterizing male and female populations are equal to each other, $d_{1}=d_{2}, a=c, b=d$, then taking a difference of equations (1.1) and (1.2), we conclude that $u=v$. In this case we can reduce this system of equations to the equation

$$
\frac{\partial u}{\partial t}=\frac{\partial^{2} u}{\partial x^{2}}+a u^{2}(1-I(u))-b u .
$$

It is a particular case of a more general nonlocal reaction-diffusion equation

$$
\frac{\partial u}{\partial t}=\frac{\partial^{2} u}{\partial x^{2}}+a u^{k}(1-J(u))-b u, \quad J(u)=\int_{-\infty}^{\infty} \phi(x-y) u(y, t) d y .
$$

Here $k=1$ corresponds to asexual and $k=2$ to sexual reproduction. It has solutions in the form of simple and periodic travelling waves and stationary pulses [2], [3], [4], [8], [10]. The case where $k=2$ and $\phi(x) \equiv 1$ corresponds to sexual reproduction with global consumption of resources. Equation (1.3) does not have travelling wave solutions but it has stable stationary pulses [7].

In this work we will study the existence of stationary solutions of system (1.1), (1.2) in the case where the parameters of male and female populations can be different from each other. In this case the system of equations cannot be reduced to the single equation and the proof of the existence of solutions becomes much more involved. We will study it by the Leray-Schauder method which is based on the topological degree for elliptic operators in unbounded domains [6]. We will carry out numerical simulations of this model in order to verify stability of pulse solutions and we will use it to describe the human height distribution.

\section{Existence of solutions}

In this section we will study the existence of stationary solutions (pulses) for the reactiondiffusion systems without the integral terms. We will use the Leray-Schauder method for the elliptic problems in unbounded domains. In order to obtain a priori estimates of solutions, we will first show that monotone and nonmonotone solutions of the corresponding problems on the half-axis are separated from each other in the norm of the function space. After that we will obtain a priori estimates of monotone solutions and will use the Leray-Schauder method only for them. This approach was developed before for travelling wave solutions [7], [9]. In the next section we will use the results of this section in order to prove the existence of stationary solutions of system (1.1), (1.2). 


\subsection{Problem depending on parameter}

In this section we will consider the problem

$$
\begin{gathered}
u^{\prime \prime}+a_{\tau} u v-b_{\tau} u=0, \quad v^{\prime \prime}+c_{\tau} u v-d_{\tau} v=0 \\
u^{\prime}(0)=v^{\prime}(0)=0, \quad u(\infty)=v(\infty)=0
\end{gathered}
$$

with the coefficients that depend on the parameter $\tau \in[0,1]$. Solutions of this problem can be extended on the whole axis by symmetry. The coefficients are positive for all values of parameter and these dependencies are continuous. We will prove the following lemma about monotonicity of solutions.

Lemma 2.1. Suppose that there exists a solution $u_{\tau}(x), v_{\tau}(x) \in C^{2+\alpha}\left(\mathbb{R}_{+}\right)$of problem (2.1), (2.2) continuous with respect to $\tau$ in the $C^{1}\left(\mathbb{R}_{+}\right)$-norm. If both components of the solution are monotonically decreasing for some $\tau_{0} \in[0,1]$, then this is also true for all other values of $\tau$.

Proof. Suppose that the assertion of the lemma does not hold, and at least one of the components of solution is not strictly decreasing for some $\tau_{1} \in[0,1]$. We can assume for certainty that $\tau_{1}>\tau_{0}$. Then there exists a value $\tau_{*}, \tau_{0} \leq \tau_{*} \leq \tau_{1}$ such that the functions $u_{\tau}(x)$ and $v_{\tau}(x)$ are monotonically decreasing for all $\tau \in\left[\tau_{0}, \tau_{*}\right)$ and at least one of these functions is not monotonically decreasing for any $\tau \in\left[\tau_{*}, \tau_{1}\right)$. We will show that this assumption will lead to a contradiction.

We will use the notation $u_{*}(x)=u_{\tau_{*}}(x), v_{*}(x)=v_{\tau_{*}}(x)$. Let us note first of all that $u_{*}^{\prime}(x) \leq 0, v_{*}^{\prime}(x) \leq 0$ for all $x>0$ since these are $C^{1}\left(\mathbb{R}_{+}\right)$limits of decreasing functions. Then the following two properties hold:

$$
u_{*}(0)>d_{\tau_{*}} / c_{\tau_{*}}, \quad v_{*}(0)>b_{\tau_{*}} / a_{\tau_{*}}
$$

and

$$
u_{*}^{\prime}(x)<0, \quad v_{*}^{\prime}(x)<0, \quad x>0 .
$$

Let us begin with the first one. Since $u_{*}(x)$ and $v_{*}(x)$ are non-increasing functions for $x \geq 0$ and $u_{*}^{\prime}(0)=v_{*}^{\prime}(0)=0$, then $u_{*}^{\prime \prime}(0) \leq 0$ and $v_{*}^{\prime \prime}(0) \leq 0$. We will show that these inequalities are strict. Set $w(x)=-u_{*}^{\prime}(x), z(x)=-v_{*}^{\prime}(x)$. Differentiating equations of system $(2.1)$, we obtain the following equations for these new functions:

$$
\begin{gathered}
w^{\prime \prime}+a_{*} v_{*}(x) w+a_{*} u_{*}(x) z-b_{*} w=0, \\
z^{\prime \prime}+c_{*} u_{*}(x) z+c_{*} v_{*}(x) w-d_{*} z=0, \\
w(0)=z(0)=0
\end{gathered}
$$


Here $a_{*}=a_{\tau_{*}}$, and similarly for the other coefficients. Let us recall that $w(x) \geq 0, z(x) \geq 0$ for all $x \geq 0$. The coefficients of this system are non-negative since $u_{*}(x) \geq 0, v_{*}(x) \geq 0$. Hence from the Hopf lemma it follows that either $w^{\prime}(0)>0$ or $w(x) \equiv 0$ and $z^{\prime}(0)>0$ or $z(x) \equiv 0$. We will prove below that these functions cannot be identically zero. Therefore $u_{*}^{\prime \prime}(0)<0, v_{*}^{\prime \prime}(0)<0$. Inequalities (2.3) follow from these ones and from equations $(2.1)$.

Similarly, from the positiveness theorem it follows that the functions $w(x)$ and $z(x)$ are strictly positive for all $x>0$ or they are identically zero. As above, we will show that they cannot be identically zero. Hence they are strictly positive, and $u_{*}^{\prime}(x)<0, v_{*}^{\prime}(x)<0$ for all $x>0$. This proves inequalities (2.4).

It remains to prove that the functions $w(x)$ and $z(x)$ are not identically zero. Suppose that $w(x) \equiv 0$. Then $u_{*}(x) \equiv$ const. Since $u_{*}(\infty)=0$, then $u(x) \equiv 0$. From the second equation in (2.1) we conclude that $v_{*}(x) \equiv 0$. Since $u_{\tau}(x) \rightarrow u_{*}(x), v_{\tau}(x) \rightarrow v_{*}(x)$ as $\tau \rightarrow \tau_{*}$ uniformly in $C^{1}$, then $u_{\tau}(0)<d_{\tau} / c_{\tau}$ and $v_{\tau}(0)<b_{\tau} / a_{\tau}$ for $\tau$ sufficiently close to $\tau_{*}$. Moreover $u_{\tau}(0)>0, v_{\tau}(0)>0$ since these functions are monotonically decreasing. From equations $(2.1)$ we conclude that $u_{\tau}^{\prime \prime}(0)>0, v_{\tau}^{\prime \prime}(0)>0$. Hence we obtain a contradiction with the assumption that these functions are decreasing.

Thus we proved that the functions $u_{*}(x)$ and $v_{*}(x)$ are not identically zero and that they satisfy $(2.3),(2.4)$.

From the definition of $\tau_{*}$ it follows that there exists a sequence $\tau_{n} \searrow \tau_{*}$ such that $u_{\tau_{n}}(x) \rightarrow$ $u_{*}(x)$ and $v_{\tau_{n}}(x) \rightarrow v_{*}(x)$ in $C^{1}\left(\mathbb{R}_{+}\right)$as $\tau_{n} \rightarrow \tau_{*}$. Denote $u_{n}(x)=u_{\tau_{n}}(x), v_{n}(x)=v_{\tau_{n}}(x)$. Then there is a sequence of positive numbers $x_{n}$ such that $u_{n}^{\prime}\left(x_{n}\right)=0$ or $v_{n}^{\prime}\left(x_{n}\right)=0$. We will show that this assumption leads to a contradiction.

Without loss of generality we can affirm that one of the following three cases holds: $x_{n} \rightarrow 0, x_{n} \rightarrow x_{*}>0, x_{n} \rightarrow \infty$. We will show that each of them leads to a contradiction.

1. $x_{n} \rightarrow 0$. Then passing to the limit we obtain $u_{*}^{\prime \prime}(0)=0$ or $v_{*}^{\prime \prime}(0)=0$. This gives a contradiction with (2.3).

2. $x_{n} \rightarrow x_{*}$. Then $u_{*}^{\prime}\left(x_{*}\right)=0$ or $v_{*}^{\prime}\left(x_{*}\right)=0$. This contradicts (2.4).

3. $x_{n} \rightarrow \infty$. Since the functions $u_{n}(x)$ and $v_{n}(x)$ converge to $u_{*}(x)$ and $v_{*}(x)$ uniformly on the half-axis together with their first derivatives, then we can choose such $\tilde{x}>0$ and $N>0$ that

$$
u_{n}^{\prime}(\tilde{x})<0, \quad v_{n}^{\prime}(\tilde{x})<0, \quad n \geq N ; \quad u_{n}(x)+v_{n}(x)<\min \left(b_{n} / a_{n}, d_{n} / c_{n}\right), \quad x \geq \tilde{x}, \quad n \geq N .
$$

We will show that

$$
u_{n}^{\prime}(x)<0, \quad v_{n}^{\prime}(x)<0, \quad x \geq \tilde{x}, \quad n \geq N .
$$

Suppose that this is not the case and for some $x_{0}>\tilde{x}, n_{0} \geq N$, at least one of the following inequalities hold: $u_{n_{0}}^{\prime}\left(x_{0}\right) \geq 0, v_{n_{0}}^{\prime}\left(x_{0}\right) \geq 0$.

Denote $w(x)=-u_{n_{0}}^{\prime}(x), z(x)=-v_{n_{0}}^{\prime}(x)$. These functions satisfy the equations 


$$
\begin{gathered}
w^{\prime \prime}+a_{n_{0}} v_{n_{0}}(x) w+a_{n_{0}} u_{n_{0}}(x) z-b_{n_{0}} w=0, \\
z^{\prime \prime}+c_{n_{0}} u_{n_{0}}(x) z+c_{n_{0}} v_{n_{0}}(x) w-d_{n_{0}} z=0 .
\end{gathered}
$$

From (2.8) we conclude that $w(\tilde{x})>0, z(\tilde{x})>0$. Further, we have at least on of the following inequalities: $w\left(x_{0}\right) \leq 0, z\left(x_{0}\right) \leq 0$. Therefore we can choose a number $h \geq 0$ such that

$$
\hat{w}(x) \equiv w(x)+h \geq 0, \quad \hat{z}(x) \equiv z(x)+h \geq 0, \quad x \geq \tilde{x},
$$

and there exists $x_{1}>\tilde{x}$ such that at least one of the following equalities hold: $\hat{w}\left(x_{1}\right)=$ $0, \hat{z}\left(x_{1}\right)=0$. Let us write the equations for the functions $\hat{w}$ and $\hat{z}$. From (2.10), (2.11) we obtain:

$$
\begin{aligned}
& \hat{w}^{\prime \prime}+a_{n_{0}} v_{n_{0}}(x)(\hat{w}-h)+a_{n_{0}} u_{n_{0}}(x)(\hat{z}-h)-b_{n_{0}}(\hat{w}-h)=0, \\
& \hat{z}^{\prime \prime}+c_{n_{0}} u_{n_{0}}(x)(\hat{z}-h)+c_{n_{0}} v_{n_{0}}(x)(\hat{w}-h)-d_{n_{0}}(\hat{z}-h)=0 .
\end{aligned}
$$

This system of equations can be written as follows:

$$
\begin{gathered}
\hat{w}^{\prime \prime}+a_{n_{0}} v_{n_{0}}(x) \hat{w}+a_{n_{0}} u_{n_{0}}(x) \hat{z}-b_{n_{0}} \hat{w}+p(x)=0, \\
\hat{z}^{\prime \prime}+c_{n_{0}} u_{n_{0}}(x) \hat{z}+c_{n_{0}} v_{n_{0}}(x) \hat{w}-d_{n_{0}} \hat{z}+q(x)=0,
\end{gathered}
$$

where

$p(x)=h\left(b_{n_{0}}-a_{n_{0}}\left(u_{n_{0}}(x)+v_{n_{0}}(x)\right)\right)>0, \quad q(x)=h\left(d_{n_{0}}-c_{n_{0}}\left(u_{n_{0}}(x)+v_{n_{0}}\right)(x)\right)>0, \quad x \geq \tilde{x}$.

These estimates hold by virtue of (2.8).

Let us recall that

$$
a_{n_{0}} u_{n_{0}}(x) \hat{z}(x) \geq 0, \quad c_{n_{0}} v_{n_{0}}(x) \hat{w}(x) \geq 0 .
$$

Hence we can apply the positiveness theorem to equations (2.14), (2.15). Since

$$
\hat{w}(x) \geq 0, \quad \hat{z}(x) \geq 0, \quad x \geq \tilde{x} ; \quad \hat{w}(\tilde{x})>0, \quad \hat{z}(\tilde{x})>0 ; \quad \hat{w}\left(x_{1}\right)=0 \quad \text { or } \quad \hat{z}\left(x_{1}\right)=0
$$

then we obtain a contradiction with the positiveness theorem at least in one of these equations.

Thus, (2.9) is proved. Hence $x_{n}$ cannot tend to infinity. This contradiction completes the proof of the lemma. 


\subsection{A priori estimates of monotone solutions}

\subsubsection{Estimate of the maximum of solution}

We consider the problem

$$
\begin{gathered}
u^{\prime \prime}+a_{\tau} u v-b_{\tau} u=0, \quad v^{\prime \prime}+c_{\tau} u v-d_{\tau} v=0, \\
u^{\prime}(0)=v^{\prime}(0)=0, \quad u(\infty)=v(\infty)=0 .
\end{gathered}
$$

By a change of variables it can be reduced to the case where $a_{\tau}=c_{\tau}=1$. Suppose that it has a monotonically decreasing solution. We will estimate its value at $x=0$. Let us begin with the particular case where $b_{\tau}=d_{\tau}$. Then $u=v$. We multiply the first equation by $u^{\prime}$ and integrate from 0 to $\infty$ :

$$
b_{\tau} u^{2}(0)=-2 \int_{0}^{\infty} u(x) u^{\prime}(x) v(x) d x=-2 \int_{0}^{\infty} u^{2}(x) u^{\prime}(x) d x .
$$

Hence $u(0)=3 b_{\tau} / 2$. If $b_{\tau} \neq d_{\tau}$, then the estimate of the maximum of the solution becomes much more involved. We present it in the Appendix.

Model with global consumption. Along with problem (2.1), (2.2), we will also consider the system of equations

$$
\begin{aligned}
& u^{\prime \prime}+a_{\tau}^{0} u v(1-I(u)-I(v))-b_{\tau} u=0, \\
& v^{\prime \prime}+c_{\tau}^{0} u v(1-I(u)-I(v))-d_{\tau} v=0
\end{aligned}
$$

on the half-axis $x>0$ with the following conditions a $x=0$ and $x \rightarrow \infty$ :

$$
u^{\prime}(0)=v^{\prime}(0)=0, \quad u(+\infty)=v(+\infty)=0 .
$$

Here

$$
I(u)=\int_{0}^{\infty} u(y) d y, \quad I(v)=\int_{0}^{\infty} v(y) d y
$$

Set

$$
a_{\tau}=a_{\tau}^{0}(1-I(u)-I(v)), \quad c_{\tau}=c_{\tau}^{0}(1-I(u)-I(v)) .
$$

The we obtain problem (2.1), (2.2). We will consider monotonically decreasing solutions $u(x), v(x) \in C^{2+\alpha}\left(\mathbb{R}_{+}\right)$.

Let us note first of all that there is the estimate $I(u)+I(v)<1$ for solutions of problem (2.19)-(2.21). Indeed, otherwise the second derivatives of solutions are positive. Since $u^{\prime}(0)=$ $v^{\prime}(0)=0$, then in the case of positive second derivatives the solution is monotonically increasing. We obtain a contradiction with the assumption that the solution is decreasing. 
Lemma 2.2. There exists a positive constant $M$ which depends on the coefficients $a_{\tau}, b_{\tau}, c_{\tau}, d_{\tau}$ such that monotone solutions $u_{t}(x), v_{\tau}(x)$ of problem (2.19)-(2.21) satisfy the estimate

$$
u_{\tau}(0) \leq M, \quad v_{\tau}(0) \leq M
$$

Proof. Denote by $x_{u}$ the solution of the equation

$$
c^{0} u(x)(1-I(u)-I(v))=d
$$

and by $x_{v}$ the solution of the equation

$$
a^{0} v(x)(1-I(u)-I(v))=b
$$

(the subscript $\tau$ is omitted). Then

$$
u^{\prime \prime}(x) \quad\left\{\begin{array}{cc}
<0, & 0 \leq x<x_{v} \\
>0, & x>x_{v}
\end{array}, \quad v^{\prime \prime}(x) \quad\left\{\begin{array}{cc}
<0, & 0 \leq x<x_{u} \\
>0, & x>x_{u}
\end{array} .\right.\right.
$$

The function $u(x)$ satisfies the problem

$$
u^{\prime \prime}-b u+f(x)=0,\left.\quad u\right|_{x=x_{v}}=u\left(x_{v}\right), \quad u(\infty)=0
$$

on the half-axis $x>x_{v}$. Here $f(x)=a^{0} u(x) v(x)(1-I(u)-I(v))>0$. Therefore it can be estimated from below by the solution of the problem

$$
u^{\prime \prime}-b u=0,\left.\quad u\right|_{x=x_{v}}=u\left(x_{v}\right), \quad u(\infty)=0 .
$$

Hence

$$
u(x) \geq u\left(x_{v}\right) e^{-b\left(x-x_{v}\right)}, \quad x \geq x_{v} .
$$

Since $I(u)<1$, then $u\left(x_{v}\right)<b$ and, by virtue of the previous inequality, $\left|u^{\prime}\left(x_{v}\right)\right|<b^{2}$.

Next, we consider the same function in the interval $0<x<x_{v}$. Since $u^{\prime \prime}(x)<0$ for these values of $x$, then $\left|u^{\prime}(x)\right|<b^{2}, 0<x<x_{v}$, and

$$
u(0)-u\left(x_{v}\right) \leq b^{2} x_{v}
$$

Hence $u(0) \leq b+b^{2} x_{v}$. On the other hand, since $u^{\prime \prime}(x)<0$ in this interval, then

$$
u(x) \geq u(0)-\frac{u(0)-u\left(x_{v}\right)}{x_{v}} x, \quad 0 \leq x \leq x_{v} .
$$

Therefore

$$
I(u)>\int_{0}^{x_{v}} u(y) d y>\frac{1}{2}\left(u(0)-u\left(x_{v}\right)\right) x_{v} .
$$

From this estimate we obtain that $u(0)<b+2 I(u) / x_{v}$. Thus 


$$
u(0) \leq \min \left(b+b^{2} x_{v}, b+2 / x_{v}\right),
$$

and $u(0)$ is bounded independently of $x_{v}$.

Similarly we can estimate $v(0)$. The lemma is proved.

\subsubsection{Estimates in weighted Hölder spaces}

Lemma 2.3. Consider monotonically decreasing solutions $u_{\tau}(x), v_{\tau}(x)$ of problem (2.16), (2.17) assuming that they are uniformly bounded in the $C^{2+\alpha}\left(\mathbb{R}_{+}\right)$-norm. Let $x_{u}$ the solution of the equation $c_{\tau} u_{\tau}(x)=d_{\tau} / 2$ and by $x_{v}$ the solution of the equation $a_{\tau} v_{\tau}(x)=b_{\tau} / 2$ Then $x_{u}$ and $x_{v}$ are bounded independently of $\tau$.

Proof. Suppose that the assertion of the lemma does not hold and at least one of these values tends to infinity as $\tau_{n} \rightarrow \tau_{0}$. Let it be $x_{u}$ and $x_{u} \geq x_{v}$. Consider the functions $w_{n}(x)=u_{\tau_{n}}\left(x+x_{u}\right)$ and $z_{n}(x)=v_{\tau_{n}}\left(x+x_{u}\right)$. Then the first one is a monotonically decreasing function defined in the half-axis $x>-x_{u}$. It satisfies the equation

$$
w^{\prime \prime}+a_{\tau_{n}} z_{n}(x) w-b_{\tau_{n}} w=0
$$

and

$$
w_{n}(0)=\frac{d_{\tau_{n}}}{2 c_{\tau_{n}}}, \quad z_{n}(0) \leq \frac{b_{\tau_{n}}}{2 a_{\tau_{n}}} .
$$

Since the functions $w_{n}(x)$ and $z_{n}(x)$ are uniformly bounded, then we can get for them uniform estimates in the Hölder norm $C^{2+\alpha}$ on the half-axis $x \geq-x_{u}$. Therefore we can choose locally convergent subsequence of these sequences,

$$
w_{n}(x) \rightarrow w_{0}(x), \quad z_{n}(x) \rightarrow z_{0}(x), \quad n \rightarrow \infty .
$$

The functions $w_{0}(x)$ and $z_{0}(x)$ are defined on the whole axis, they are non-increasing, and they are bounded and continuous together with their second derivatives. Passing to the limit in equation (2.23), we get the equality

$$
w_{0}^{\prime \prime}+a_{\tau_{0}} z_{0}(x) w_{0}-b_{\tau_{0}} w_{0}=0, \quad x \in \mathbb{R}
$$

and

$$
w_{0}(0)=\frac{d_{\tau_{0}}}{2 c_{\tau_{0}}}, \quad z_{0}(0) \leq \frac{b_{\tau_{0}}}{2 a_{\tau_{0}}} .
$$

Moreover, $w_{0}(\infty)=0, w_{0}(-\infty)=w_{-}>0$. The limits $w_{0}(-\infty)=w_{-}$and $z_{0}(-\infty)=z_{-}$ should satisfy the equation

$$
\left(a_{\tau_{0}} z_{-}-b_{\tau_{0}}\right) w_{-}=0
$$


since $w_{0}^{\prime \prime}(x) \rightarrow 0$ as $x \rightarrow-\infty$. As $w_{-}>0$, then $a_{\tau_{0}} z_{-}-b_{\tau_{0}}=0$ and

$$
a_{\tau_{0}} z_{0}(x)-b_{\tau_{0}} \leq 0, \quad x \in \mathbb{R}
$$

because $z_{0}(x)$ is a non-increasing function. From this inequality and (2.24) it follows that $w_{0}^{\prime \prime}(x) \geq 0$ for all $x \in \mathbb{R}$. Therefore $w_{0}(x)$ can be a bounded function only if $w_{0}^{\prime \prime}(x) \equiv 0$ for all $x$. Hence

$$
a_{\tau_{0}} z_{0}(x)-b_{\tau_{0}} \equiv 0, \quad x \in \mathbb{R} .
$$

This equality contradicts (2.25). The lemma is proved.

Let $\mu(x)=1+x^{2}$. Consider the weighted Hölder space $C_{\mu}^{2+\alpha}\left(\mathbb{R}_{+}\right), 0<\alpha<1$ with the norm

$$
\|u\|_{C_{\mu}^{2+\alpha}\left(\mathbb{R}_{+}\right)}=\|u \mu\|_{C^{2+\alpha}\left(\mathbb{R}_{+}\right)} .
$$

Lemma 2.4. Monotonically decreasing solutions $u_{\tau}(x), v_{\tau}(x)$ of problem (2.1), (2.2) are bounded in the norm $C_{\mu}^{2+\alpha}\left(\mathbb{R}_{+}\right)$independently of $\tau$.

Proof. It is sufficient to estimate the uniform norm of the functions $u_{\tau}(x) \mu(x), v_{\tau}(x) \mu(x)$. Then the estimates of the Hölder norm will follow by virtue of the equations. In order to estimate these functions, it is sufficient to note that they are uniformly exponentially decreasing for $x \geq x_{0}=\max \left(x_{u}, x_{v}\right)$, where these values are defined in Lemma 2.3. Therefore the functions are bounded for $x \geq x_{0}$. On the other hand, since $x_{0}$ is bounded independently of $\tau$ and the functions $u_{\tau}(x), v_{\tau}(x)$ are also bounded independently of $\tau$, then the functions $u_{\tau}(x) \mu(x), v_{\tau}(x) \mu(x)$ are bounded independently of $\tau$ in the interval $0 \leq x \leq x_{0}$. The lemma is proved.

Remark 2.5. The assertion of the lemma is also valid for problem (2.19)-(2.21). The proof of the lemma in this case is similar.

\subsection{Leray-Schauder method}

We will prove existence of solutions by the Leray-Schauder method. Consider the operator $A_{\tau}: E \rightarrow F$, where

$$
\begin{gathered}
A_{\tau}\left(\begin{array}{c}
u \\
v
\end{array}\right)=\left\{\begin{array}{c}
u^{\prime \prime}+a_{\tau} u v-b_{\tau} u \\
v^{\prime \prime}+c_{\tau} u v-d_{\tau} v
\end{array}\right. \\
E=\left\{u, v \in C_{\mu}^{2+\alpha}\left(\mathbb{R}_{+}\right), u^{\prime}(0)=v^{\prime}(0)=0\right\}, \quad F=C_{\mu}^{\alpha}\left(\mathbb{R}_{+}\right) .
\end{gathered}
$$

This operator is bounded and continuous. Assuming that the coefficients $a_{\tau}, b_{\tau}, c_{\tau}, d_{\tau}$ are sufficiently smooth functions of $\tau$, we affirm that this operator is proper on closed bounded sets and topological degree can be defined for it [6]. 
From the results of the previous section it follows that all monotone solutions of the equation $A_{\tau}=0$ are strictly inside of some ball $B_{R}$ of the space $E$. Moreover from the results of Section 2.1 we conclude that there exists a positive constant $\epsilon$ such that

$$
\left\|u_{M}-u_{N}\right\|_{E}+\left\|v_{M}-v_{N}\right\|_{E} \geq \epsilon, \quad \forall \tau \in[0,1] .
$$

for any monotone solution $u_{M}, v_{M}$ and any non-monotone solution $u_{N}, v_{N}$. Since the set of solutions is compact inside the ball $B_{R}$ by virtue of the properness of the operator, then it is possible to construct it is possible construct a domain $D \subset B_{R}$ which contains all monotone solutions and which does not contain any non-monotone solution. Indeed, it is sufficient to take a ball of the radius $\epsilon / 2$ around each monotone solution, to take their union and choose its finite covering.

Let us also note that if $\epsilon$ is sufficiently small, then domain $D$ does not contain the trivial solution. Indeed, all monotonically decreasing solutions satisfy the inequalities $u(0) \geq$ $d_{\tau} / c_{\tau}, v(0) \geq b_{\tau} / a_{\tau}$.

We can apply the Leray-Schauder method to the operator $A_{\tau}$ in the domain $D$. We will obtain the existence of solutions if the topological degree $\gamma\left(A_{\tau}, D\right)$ is different from 0 for some value of $\tau$. In the other words, it is sufficient to construct a model operator with the degree different from 0 .

\subsection{Model problem}

Consider the problem

$$
u^{\prime \prime}+u v-b u=0, \quad v^{\prime \prime}+u v-b v=0
$$

on the half-axis $x>0$ with conditions

$$
u^{\prime}(0)=v^{\prime}(0)=0, \quad u(\infty)=v(\infty)=0 .
$$

Taking a difference of the two equations in (2.26) we can conclude that $u(x) \equiv v(x)$. Therefore we reduce problem $(2.26),(2.27)$ to the scalar problem

$$
u^{\prime \prime}+u^{2}-b u=0, \quad u^{\prime}(0)=0, \quad u(\infty)=0 .
$$

It can be easily verified that it has a unique monotonically decreasing solutions for any $b>0$. Denote this solution by $u_{0}$ and consider problem (2.26) linearized about this solution:

$$
\begin{gathered}
\tilde{u}^{\prime \prime}+\tilde{u} v_{0}(x)+\tilde{v} u_{0}(x)-b \tilde{u}=0, \\
\tilde{v}^{\prime \prime}+\tilde{u} v_{0}(x)+\tilde{v} u_{0}(x)-b \tilde{v}=0, \\
\tilde{u}^{\prime}(0)=\tilde{v}^{\prime}(0)=0, \quad \tilde{u}(\infty)=\tilde{v}(\infty)=0 .
\end{gathered}
$$


Here $u_{0}(x) \equiv v_{0}(x)$. Taking a difference of equations (2.29) and (2.30), we verify that $\tilde{u}(x) \equiv \tilde{v}(x)$. Hence we can reduce this problem to the following one:

$$
\begin{aligned}
& \tilde{u}^{\prime \prime}+2 \tilde{u} u_{0}(x)-b \tilde{u}=0, \\
& \tilde{u}^{\prime}(0)=0, \quad \tilde{u}(\infty)=0 .
\end{aligned}
$$

This is exactly the problem obtained as linearization of (2.28). It can be proved that this problem has only trivial solution [7]. Therefore we can calculate the index of this stationary point, that is topological degree with respect to small ball around this point:

$$
\text { ind } u_{0}=(-1)^{\nu}
$$

where $\nu$ is the number of positive eigenvalues of the linearized operator together with their multiplicities. Since 0 is not an eigenvalue of this operator, then the index is well defined and it is different from zero.

\subsection{Existence theorem}

Consider the problem

$$
\begin{gathered}
u^{\prime \prime}+a u v-b u=0, \quad v^{\prime \prime}+c u v-d v=0 \\
u(0)=v(0)=0, \quad u(\infty)=v(\infty)=0 .
\end{gathered}
$$

Set $u=w / c, v=z / a$. Then the new functions $w$ and $z$ satisfy the problem

$$
\begin{aligned}
& w^{\prime \prime}+w z-b w=0, \quad z^{\prime \prime}+w z-d z=0 \\
& w(0)=z(0)=0, \quad w(\infty)=z(\infty)=0 .
\end{aligned}
$$

We can now formulate the main result of this section.

Theorem 2.6. Problem (2.36), (2.37) has a monotonically decreasing solution for any positive numbers $b$ and $d$.

Proof. We set $a_{\tau}=c_{\tau}=1, b_{\tau}=b, d_{\tau}=\tau b+(1-\tau) d, \tau \in[0,1]$ and consider the operator $A_{\tau}$ defined in Section 2.3. A priori estimates of monotone solutions of the equation $A_{\tau}=0$ are obtained in Section 2.2. We can construct a domain $D \subset E$ such that it contains only monotonically decreasing solutions of this equation and it does not contain non-monotone solutions and the trivial solution (Sections 2.1, 2.3). The model problem is studied in Section 2.4. Since $\gamma\left(A_{\tau}, D\right) \neq 0$ for $\tau=1$, then this is also true for $\tau=0$. From this we conclude about the existence of solutions for $\tau=0$. 


\section{System with global consumption}

\subsection{Existence of solutions}

Consider the problem

$$
\begin{gathered}
u^{\prime \prime}+\operatorname{auv}(1-I(u)-I(v))-b u=0, \quad v^{\prime \prime}+\operatorname{cuv}(1-I(u)-I(v))-d v=0 \\
u(0)=v(0)=0, \quad u(\infty)=v(\infty)=0 .
\end{gathered}
$$

Set $k=1-I(u)-I(v), u=w / c k, v=z / a k$. Then the new functions $w$ and $z$ satisfy the problem

$$
\begin{aligned}
& w^{\prime \prime}+w z-b w=0, \quad z^{\prime \prime}+w z-d z=0 \\
& w(0)=z(0)=0, \quad w(\infty)=z(\infty)=0 .
\end{aligned}
$$

Existence of solutions of this problem is proved in Theorem 2.6. Denote by $w_{0}(x), z_{0}(x)$ a solution of this problem. Then we have the equality

$$
k=1-\frac{1}{c k} I\left(w_{0}\right)-\frac{1}{a k} I\left(z_{0}\right) .
$$

Hence

$$
k^{2}-k+\beta=0
$$

where $\beta=I\left(w_{0}\right) / c+I\left(z_{0}\right) / a$. This equation has two positive solutions if $\beta<1 / 4$ no solutions for $\beta>1 / 4$. Therefore we have the following existence result.

Theorem 3.1. For any solution $w_{0}(x), z_{0}(x)$ of problem (3.3), (3.4), problem (3.1), (3.2) has two solutions $u_{1,2}(x)=w_{0}(x) / c k_{1,2}, v_{1,2}(x)=z_{0}(x) / a k_{1,2}$ if $I\left(w_{0}\right) / c+I\left(z_{0}\right) / a<1 / 4$. Here $k_{1,2}$ are solutions of equation (3.5). There is only one such solution in the case of equality and no solutions if the inequality is opposite.

We prove here the existence of solutions of problem (3.1), (3.2) with global consumption of resources reducing it to the reaction-diffusion system with constant coefficients (without integral terms). On the other hand, we have obtained above a priori estimates of solutions directly for problem (3.1), (3.2), and we can apply for it the Leray-Schauder method. However, as it follows from the previous theorem, the number of solutions of this problem is even, and the total value of the topological degree equals zero. Therefore we cannot directly conclude that there exist solutions of problem (3.1), (3.2), and we reduce it to problem (3.3), $(3.4)$. 


\subsection{Parents with different phenotype}

In the previous section we studied existence of solutions of problem (3.1), (3.2) on the halfaxis. It can be extended by symmetry on the whole axis. Hence we have the existence of positive solutions of the problem

$$
\begin{gathered}
u^{\prime \prime}+a u v(1-I(u)-I(v))-b u=0, \quad v^{\prime \prime}+c u v(1-I(u)-I(v))-d v=0, \\
u( \pm \infty)=v( \pm \infty)=0 .
\end{gathered}
$$

Let us note that these solutions are invariant with respect to translation in space.

We will briefly discuss biological aspects of this model. The reproduction terms in equations (3.6) are proportional to the product $u(x) v(x)$ of the densities of males and females. We take these densities at the same space point $x$. If we interpret the space variable as phenotype of the individuals, then this means that both parents have the same phenotype, and their offsprings have the same phenotype as parents with a small perturbation due to random mutations (diffusion term). However the phenotypes of parents can be different. For example, it is known that there is a relation between the sizes of males and females who form a couple. In order to take this difference into account, we will replace the term $u(x) v(x)$ by the term $u(x+h) v(x)$ assuming that the phenotype of males has a constant shift with respect to the phenotype of females. We will also assume that male offsprings have the same phenotype as their father, while female offspring as their mother. Then we get the system of equations

$$
\begin{gathered}
u(x+h)^{\prime \prime}+a u(x+h) v(x)(1-I(u)-I(v))-b u(x+h)=0, \\
v(x)^{\prime \prime}+c u(x+h) v(x)(1-I(u)-I(v))-d v(x)=0 .
\end{gathered}
$$

This system can be reduced to the system with the same phenotypes of parents if we introduce the new functions $w(x)=u(x+h), z(x)=v(x)$. Hence the result on the existence of solutions remains valid in the case of different phenotypes of males and females.

\subsection{Dynamics of solutions}

We now consider the evolution problem on the whole axis,

$$
\begin{aligned}
& \frac{\partial u}{\partial t}=d_{1} \frac{\partial^{2} u}{\partial x^{2}}+\operatorname{auv}(1-r I(u)-r I(v))-b u, \\
& \frac{\partial v}{\partial t}=d_{2} \frac{\partial^{2} v}{\partial x^{2}}+c u v(1-r I(u)-r I(v))-d v,
\end{aligned}
$$

where 


$$
I(u)=\int_{-\infty}^{\infty} u(y, t) d y, \quad I(v)=\int_{-\infty}^{\infty} v(y, t) d y
$$

We will look for solutions of this problem decaying at infinity. Theorem 3.1 provides existence of stationary solutions of this problem if we extend them on the whole axis by symmetry. If $r=0$, then these stationary solutions are unstable because the principal eigenvalue of the corresponding linearized problem is positive [9].

We carry our numerical simulations of system (3.8), (3.9) on a sufficiently large interval where the influence of the boundary can be neglected. We use the implicit finite difference method with Thomas algorithm. Numerical simulations show that the solution of system (3.8), (3.9) with $r>0$ converges to the stationary solution (pulse). From this point of view we can suppose that the pulse solution becomes stable due to the presence of the integral terms in the equations. Similarly, in the case of the scalar equation, pulse solutions are unstable. However they become stable in the case of global consumption [2], [7].

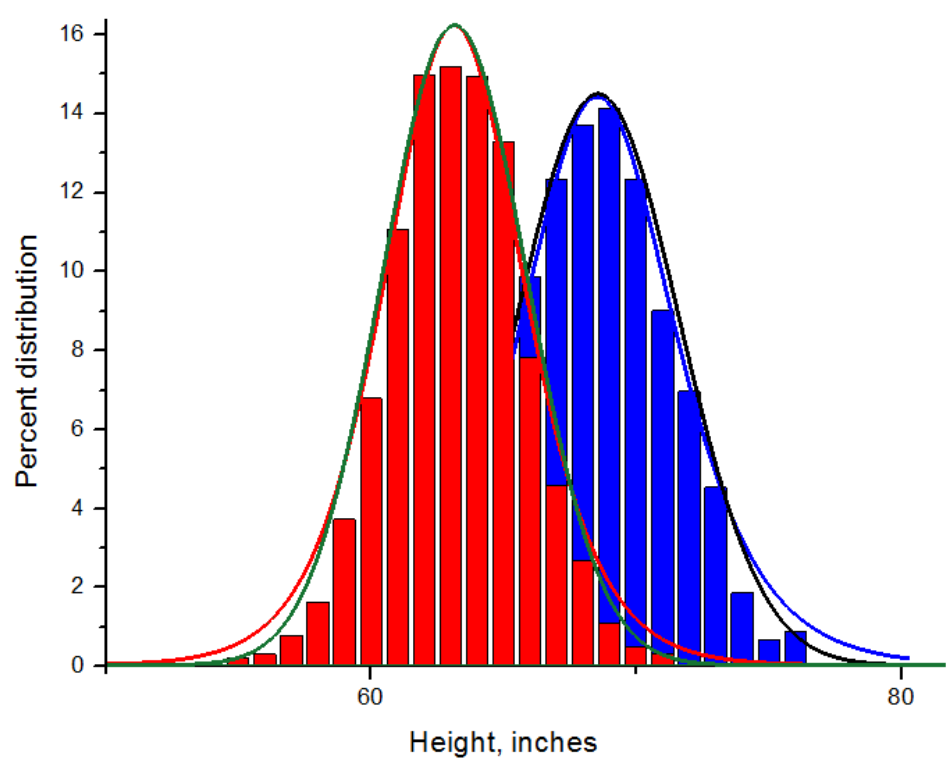

Figure 1: Human height distribution in statistical data (histograms), numerical simulations of system (3.8), (3.9) with different phenotypes of parents (cf. Section 3.2) and normal distributions (see the explanation in the text).

We will apply this system to model human height distribution. Here $x$ is the height of individuals, $u(x, t)$ is the height density distribution for men and $v(x, t)$ for women. Since the average height of men and women who form a couple are different, we will consider shifted phenotypes (heights) as described in Section 3.2:

$$
\frac{\partial u(x+h, t)}{\partial t}=d_{1} \frac{\partial^{2} u(x+h, t)}{\partial x^{2}}+a u(x+h, t) v(x, t)(1-r I(u)-r I(v))-b u(x+h, t),
$$




$$
\frac{\partial v(x, t)}{\partial t}=d_{2} \frac{\partial^{2} v(x, t)}{\partial x^{2}}+c u(x+h, t) v(x, t)(1-r I(u)-r I(v))-d v(x, t) .
$$

We will compare numerical simulations of this model with statistical data [1] and with the normal distribution. The histograms in Figure 1 show statistical data for the men height distribution (blue) and for the women height distribution (red).

Upper curves for each distribution (blue and red) show the results of numerical simulation of system (3.10), (3.11) with the value of parameters $d_{1}=3.7, a=50, r=0.393, b=1, d_{2}=$ $2.2, c=50, d=1$. We suppose that all coefficients in the first equation are the same as in the second equation except for the diffusion coefficients. In the other words, we assume that natality and mortality of men and women are the same as well as the consumption of resources. Diffusion terms show genetic variability of children with respect with their parents. Genetic variability of men is greater than that of women, and their distribution is wider.

The lower curves in both distributions correspond to the normal distributions. Black curve (men height distribution) shows the normal distribution with parameters: mean 69, standard deviation 2.8, variance 7.84; green curve (women height distribution) corresponds to the normal distribution with parameters: mean 63.6, standard deviation 2.5, variance 6.25. Model (3.8), (3.9) gives very close results to the normal distribution with some difference at the tails of the distributions. Thus, stationary solutions of system (3.8), (3.9) give a good description of the human height distribution.

\section{References}

[1] S. Abraham. Weight and height of adults 18-74 years of age, United States, 1971-74. Vital and health statistics: Series 11, Data from the National Health Survey; no. 211. DHEW publication; (PHS) 79-1659.

[2] N. Bessonov, N. Reinberg, V. Volpert. Mathematics of Darwin's diagram. Math. Model. Nat. Phenom., 9 (2014), no. 3, 5-25.

[3] S. Genieys, V. Volpert, P. Auger. Pattern and waves for a model in population dynamics with nonlocal consumption of resources. Math. Model. Nat. Phenom., 1 (2006), no. 1 , 65-82.

[4] S.A. Gourley, M.A.J. Chaplain, F.A. Davidson. Spatio-temporal pattern formation in a nonlocal reaction-diffusion equation. Dynamical systems, 16 (2001), no. 2, 173-192.

[5] Y. Nec, M. J. Ward. The stability and slow dynamics of two-spike patterns for a class of reaction-diffusion system. Math. Model. Nat. Phenom., 8 (2013), no.5.

[6] V. Volpert. Elliptic partial differential equations. Volume 1. Fredholm theory of elliptic problems in unbounded domains. Birkhäuser, 2011. 
[7] V. Volpert. Elliptic partial differential equations. Volume 2. Reaction-diffusion equations. Birkhäuser, 2014.

[8] V. Volpert, S. Petrovskii. Reaction-diffusion waves in biology. Physics of Life Reviews, 6 (2009), $267-310$.

[9] A. Volpert, Vit. Volpert, Vl. Volpert. Traveling wave solutions of parabolic systems. Translation of Mathematical Monographs, Vol. 140, Amer. Math. Society, Providence, 1994.

[10] V. Volpert, V. Vougalter. Emergence and propagation of patterns in nonlocal reactiondiffusion equations arising in the theory of speciation. In: Dispersal, individual movement and spatial ecology. M. Lewis, Ph. Maini, S. Petrovskii. Editors. Springer Applied Interdisciplinary Mathematics Series. Lecture Notes in Mathematics, Volume 2071, 2013, 331-353.

[11] V. Volpert, V. Vougalter. Existence of stationary pulses for nonlocal reaction-diffusion equations. Documenta Mathematica, 2015, in press.

\section{Appendix. Estimate of the maximum}

Consider the problem

$$
\begin{aligned}
& u^{\prime \prime}+u v-b u=0, \quad v^{\prime \prime}+u v-d v=0 \\
& u^{\prime}(0)=v^{\prime}(0)=0, \quad u(\infty)=v(\infty)=0 .
\end{aligned}
$$

Multiplying the first equation by $u^{\prime}$, the second by $v^{\prime}$ and integrating, we get

$$
b u^{2}(0)=-2 \int_{0}^{\infty} u(x) u^{\prime}(x) v(x) d x, \quad d v^{2}(0)=-2 \int_{0}^{\infty} u(x) v(x) v^{\prime}(x) d x .
$$

We can introduce the function $V(u)$ such that $v(x)=V(u(x))$ and the inverse function $U(v)$ such that $u(x)=U(v(x))$. Then the last equalities can be written in the following form:

$$
b u^{2}(0)=2 \int_{0}^{u(0)} u V(u) d u, \quad d v^{2}(0)=2 \int_{0}^{v(0)} U(v) v d v .
$$

The estimate of $u(0)$ and $v(0)$ requires several steps.

1. Suppose that $u(0)+v(0)>b+d+2 \epsilon$ for some $\epsilon>0$. Then for some $x$, either $u(x)=d+\epsilon$ or $v(x)=b+\epsilon$. Indeed, if we assume that for all $x>0, u(x)<d+\epsilon$ and $v(x)<b+\epsilon$, then this is also true for $x=0$ and we obtain a contradiction.

We will assume for certainty that $u\left(x_{0}\right)=d+\epsilon, v\left(x_{0}\right) \leq b+\epsilon$ for some $x=x_{0}>0$. Since $u(x)$ and $v(x)$ are decreasing functions, then $u(x)>d+\epsilon$ for $v>v\left(x_{0}\right)$. Therefore 


$$
d v^{2}(0)>2(d+\epsilon) \int_{v\left(x_{0}\right)}^{v(0)} v d v=(d+\epsilon)\left(v^{2}(0)-v^{2}\left(x_{0}\right)\right)
$$

Hence

$$
v(0)<v\left(x_{0}\right) \sqrt{1+d / \epsilon} \leq(b+\epsilon) \sqrt{1+d / \epsilon} \quad\left(\equiv v_{1}\right) .
$$

2. Suppose that $U(v)>k(v-\delta)$ for all $\delta \leq v \leq v(0)$ and for some $k>0, \delta>0$. Then

$$
d v^{2}(0)>2 k \int_{\delta}^{v(0)} v(v-\delta) d v=\left(\frac{2}{3} k v^{3}-k \delta v^{2}\right)_{\delta}^{v(0)}=\frac{2}{3} k\left(v^{3}(0)-\delta^{3}\right)-k \delta\left(v^{2}(0)-\delta^{2}\right)
$$

or

$$
3(d / k+\delta) v^{2}(0)>2 v^{3}(0)+\delta^{3} \quad \Rightarrow 3(d / k+\delta) v^{2}(0)>2 v^{3}(0) \quad \Rightarrow v(0)<\frac{3}{2}(d / k+\delta) .
$$

Hence for $k$ sufficiently large and $\delta$ sufficiently small we obtain that $v(0)<b$. However, this inequality should be opposite since $u^{\prime \prime}(0)<0$.

Hence there exist some values $k$ and $\delta$ such that $U\left(v_{2}\right)=k\left(v_{2}-\delta\right)$ for some $v_{2} \geq \delta$ (Figure 2). Moreover, $v_{2} \leq v(0) \leq v_{1}$. Then for all $u \geq U\left(v_{2}\right)$, we have $V(u) \geq \delta$.

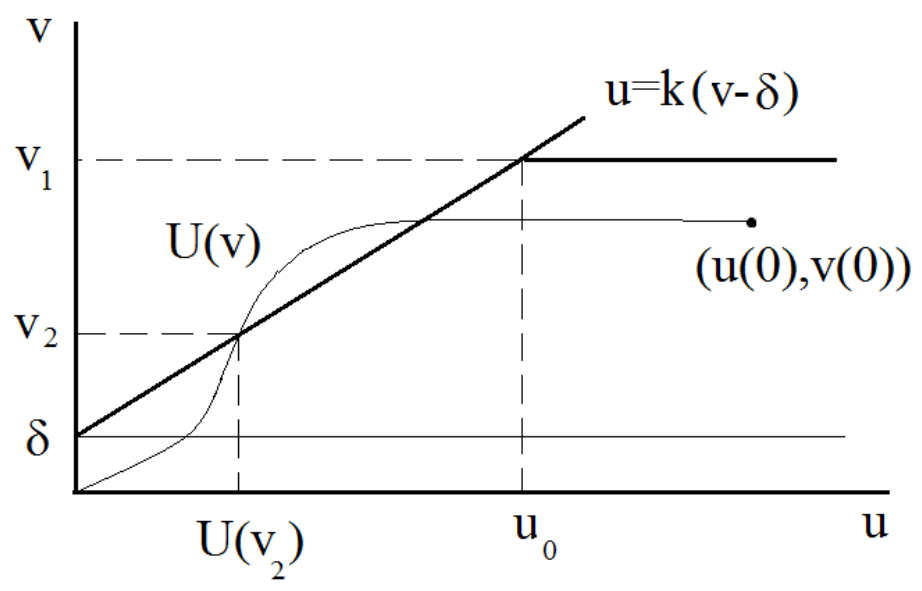

Figure 2: Illustration to the estimates of $u(0), v(0)$. The function $V(u)$ (or $U(v)$ ) is shown on the $(u, v)$-plane. It is monotonically increasing, bounded from above by $v_{1}$ and intersects the line $u=k(v-\delta)$.

3. Let $f(v)=k(v-\delta)$. Consider the inverse function $g(u)=\frac{1}{k} u+\delta$. Let $u_{0}$ be the solution of the equation 


$$
\frac{1}{k} u+\delta=v_{1}
$$

Then $u_{0}=k\left(v_{1}-\delta\right)=k((b+\epsilon) \sqrt{1+d / \epsilon}-\delta)$. For $k$ sufficiently large and $\delta$ sufficiently small, we have that $u_{0}>2 d$. Since $v_{2} \leq v_{1}$, then $U\left(v_{2}\right)=k\left(v_{2}-\delta\right) \leq k\left(v_{1}-\delta\right)=u_{0}$.

4. If we assume that $u(0)>u_{0}$, then for some $x_{1}>0$, we get $u\left(x_{1}\right)=u_{0}$. Then for all $0 \leq x \leq x_{1}, u(x) \geq u_{0}>2 d$. Since $u(x) \geq u_{0} \geq U\left(v_{2}\right)$, then $v(x) \geq v_{2} \geq \delta$ and $v(x)<v(0)<v_{1}$.

Thus, we can summarize. There are some values $x_{1}$ and $u_{0}$ such that $u\left(x_{1}\right)=u_{0}$ and

$$
2 d<u(x), \quad \delta \leq v(x) \quad 0 \leq x \leq x_{1} ; \quad v(x)<v_{1}, \quad 0 \leq x<\infty .
$$

5. From estimates (4.4) and equations (4.1) we obtain:

$$
\begin{gathered}
u\left(x_{1}\right)=u_{0}, \\
u^{\prime \prime}+p(x) u=0, \quad 0 \leq x \leq x_{1},
\end{gathered}
$$

where $p(x)=v(x)-b,|v(x)-b|<v_{1}+b \equiv q$ (notation),

$$
\begin{gathered}
\left|u^{\prime \prime}(x)\right|=|p(x)| u(x) \leq q u_{0}, \quad x \geq x_{1}, \\
v^{\prime \prime}(x)=(d-u(x)) v(x)<-d v(x) \leq-d \delta, \quad 0 \leq x \leq x_{1}
\end{gathered}
$$

Consider the system where the coefficients depend on a parameter $\tau \in[0,1]$. We suppose that the coefficients $b_{\tau}, d_{\tau}$ are uniformly bounded. Suppose that there is a sequence of solutions $u_{\tau}(x), v_{\tau}(x)$ such that $u_{\tau}(0) \rightarrow \infty$ as $\tau \rightarrow \tau_{0}, v_{\tau}(0)$ satisfies estimate (4.3). All values $\epsilon, \delta, x_{1}, u_{0}, q$ can be chosen independently of $\tau$.

Suppose that the value $x_{1}$ and the derivative $u_{\tau}^{\prime}\left(x_{1}\right)$ are uniformly bounded. Since $u_{\tau}(x)$ satisfies equation (4.6) with uniformly bounded coefficients, then $u_{\tau}(0)$ is also uniformly bounded. If this derivative tends to infinity, then from (4.7) it follows that $u_{\tau}(x)$ becomes negative. Finally, if $x_{1}$ tends to infinity, then $v_{\tau}(x)$ becomes negative since $v_{\tau}(0)$ is uniformly bounded and its second derivative satisfies (4.8). 